\title{
Parallel spin stripes and their coexistence with superconducting ground states at optimal and high doping in $\mathrm{La}_{1.6-x} \mathrm{Nd}_{0.4} \mathrm{Sr}_{x} \mathrm{CuO}_{4}$
}

\author{
Qianli Ma $\odot,{ }^{1, *}$ Kirrily C. Rule $\odot,{ }^{2}$ Zachary W. Cronkwright, ${ }^{1}$ Mirela Dragomir $\odot,{ }^{3,4}$ Gabrielle Mitchell, ${ }^{1}$ \\ Evan M. Smith $\odot,{ }^{1}$ Songxue Chi, ${ }^{5}$ Alexander I. Kolesnikov $\odot,{ }^{5}$ Matthew B. Stone $\odot,{ }^{5}$ and Bruce D. Gaulin ${ }^{1,4,6}$ \\ ${ }^{1}$ Department of Physics and Astronomy, McMaster University, Hamilton, Ontario, Canada L8S 4M1 \\ ${ }^{2}$ Australian Nuclear Science and Technology Organisation, Locked Bag 2001, Kirrawee DC, New South Wales 2232, Australia \\ ${ }^{3}$ Department of Chemistry and Chemical Biology, McMaster University, Hamilton, Ontario, Canada, L8S 4M1 \\ ${ }^{4}$ Brockhouse Institute for Materials Research, Hamilton, Ontario, Canada L8S 4M1 \\ ${ }^{5}$ Neutron Scattering Division, Oak Ridge National Laboratory, Oak Ridge, Tennessee 37830, USA \\ ${ }^{6}$ Canadian Institute for Advanced Research, MaRS Centre, West Tower, 661 University Avenue, Suite 505, Toronto, Ontario, Canada M5G 1M1
}

(Received 2 September 2020; revised 12 March 2021; accepted 29 April 2021; published 27 May 2021)

\begin{abstract}
Three-dimensional, commensurate long-range magnetic order in $\mathrm{La}_{2} \mathrm{CuO}_{4}$ quickly evolves to quasi-twodimensional, incommensurate correlations upon doping with mobile holes, and superconductivity follows for $x$ as small as 0.05 in the $\mathrm{La}_{2-x} \mathrm{Sr}_{x} / \mathrm{Ba}_{x} \mathrm{CuO}_{4}$ family of superconductors. The onset of superconductivity in these systems is known to be coincident with a remarkable rotation of the incommensurate spin order from "diagonal stripes" below $x=0.05$ to "parallel stripes" above. However, little is known about the spin correlations at optimal and high doping levels, around and beyond the proposed quantum critical point for the pseudogap phase, $p^{*}$. Here, we present elastic and inelastic neutron scattering measurements on single crystals of $\mathrm{La}_{1.6-x} \mathrm{Nd}_{0.4} \mathrm{Sr}_{x} \mathrm{CuO}_{4}$ with $x=0.125,0.19,0.24$, and 0.26 and show that two-dimensional, quasistatic, parallel spin stripes have an onset at temperatures such that the parallel spin stripe phase extends beyond $p^{*}$ and envelops the entirety of superconducting ground states in this system. We also show that the elastic order parameter for parallel spin stripes at optimum doping, $x=0.19$, displays an inflection point at superconducting $T_{c}$, while the lowenergy dynamic spectral weight of parallel stripe fluctuations grows with decreasing temperature and saturates below $T_{c}$.
\end{abstract}

DOI: 10.1103/PhysRevResearch.3.023151

\section{INTRODUCTION}

The microscopic mechanism underlying high-temperature superconductivity in copper oxide materials has been hotly debated since its original discovery in 1986 [1,2], through to the present day [3-16]. The magnetism associated with an approximately two dimensional square lattice of $\mathrm{CuO}_{2}$ has figured prominently in this debate, both as a mechanism for superconducting pairing between electrons $[10,12,14,17]$ and as a possible competing ground state $[18,19]$.

Hole-doped high- $T_{c}$ cuprates, such as $\mathrm{La}_{2-x} \mathrm{Sr}_{x} \mathrm{CuO}_{4}$ (LSCO), $\mathrm{La}_{2-x} \mathrm{Ba}_{x} \mathrm{CuO}_{4}$ (LBCO), and $\mathrm{La}_{1.6-x} \mathrm{Nd}_{0.4} \mathrm{Sr}_{x} \mathrm{CuO}_{4}$ (Nd-LSCO), are in some ways the ultimate quantum materials [20]. They display bulk superconductivity, and their parent compound, $\mathrm{La}_{2} \mathrm{CuO}_{4}$, is a stacked, two-dimensional quantum magnetic insulator, based on $S=1 / 2 \mathrm{Cu}^{2+}$ spin degrees of freedom. $\mathrm{La}_{2} \mathrm{CuO}_{4}$ orders near $300 \mathrm{~K}$ into a threedimensional (3D) commensurate $(\mathrm{C})$ antiferromagnetic $(\mathrm{AF})$

\footnotetext{
*maq1@mcmaster.ca

Published by the American Physical Society under the terms of the Creative Commons Attribution 4.0 International license. Further distribution of this work must maintain attribution to the author(s) and the published article's title, journal citation, and DOI.
}

structure $[21,22]$. However, this $3 \mathrm{D}$ C AF order is very unstable in the presence of mobile holes, such as are introduced either by substitution of $\mathrm{Sr}^{2+}$ for $\mathrm{La}^{3+}$ in $\mathrm{LSCO}$ and $\mathrm{Nd}-$ LSCO or by substitution of $\mathrm{Ba}^{2+}$ for $\mathrm{La}^{3+}$ in LBCO. The 3D $\mathrm{C}$ AF order is destroyed by $x \sim 0.02$, replaced by quasi-2D incommensurate (IC) order with much lower onset temperatures [21,21-25].

At low dopings, for $x<0.05$, the quasi-2D IC structure corresponds to "diagonal stripes" wherein local regions of $\pi-\pi$ antiferromagnetism are partitioned into domains which are finite along $(1,1,0)_{\text {tetragonal }}$ directions, which is at an angle of $45^{\circ}$ to the $\mathrm{Cu}-\mathrm{O}$ bonds within the $2 \mathrm{D}$ plane $[22,26]$. These domains are separated by domain walls running along these diagonal directions, where the excess holes reside. The domain walls introduce a $\pi$ phase shift into the AF structure, thereby giving rise to IC antiferromagnetism. While such magnetic structures do not display true long-range order, they do possess in-plane correlation lengths over tens of unit cells and are quasistatic, such that Bragg-peak-like features, well defined in $\boldsymbol{Q}$ and elastic on the time scale of neutron scattering $\left(\sim 10^{-10} \mathrm{~s}\right)$, are easily observed.

Wakimoto et al. made the remarkable observation in LSCO that the 2D IC AF structure rotates by $45^{\circ}$ at $x=0.05$ [27], exactly where superconducting ground states are first observed as a function of $x$. The same rotation [28] of the spin stripe structure was later observed in LBCO at the same 
doping level, $x$, indicating that such a rotation of the 2D IC AF structure is a general feature of these single-layer cuprate systems. The rotated stripes are referred to as "parallel" spin stripes, as the hole-bearing domain walls are oriented along

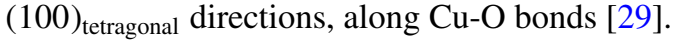

On increasing the hole doping, the IC wave vector associated with the spin stripe structure increases $\propto x$, following what is known as the Yamada relation [30]. This has the physical interpretation that the quasi-1D hole-bearing domain walls get closer together to allow for an increased hole density. The onset temperature of this 2D IC AF order also rises strongly to a maximum near $x=0.125$ [31,32]. However, what happens at dopings beyond $x=0.125$ is less clear. This is the case for at least two reasons: First, the large single crystals necessary for definitive neutron scattering experiments are progressively more difficult to grow with increasing $x$. Second, the 2D magnetic signal becomes progressively weaker and spread out in $\boldsymbol{Q}$, due to smaller ordered moments and shorter correlation lengths associated with the the increased hole density.

This paper focuses on neutron scattering studies of the Nd-LSCO system. Muon-spin rotation $(\mu \mathrm{SR})$ and nuclear magnetic resonance (NMR) techniques are sensitive to local magnetism on time scales much longer than those of neutron scattering $\left(\sim 10^{-8} \mathrm{~s}\right.$ for $\mu \mathrm{SR}$ and $\sim 10^{-6} \mathrm{~s}$ for NMR), and such studies have also been carried out on Nd-LSCO and other single-layer, hole-doped cuprates [33-35]. While the energy scale for typical neutron diffraction $(\sim 1 \mathrm{meV})$ implies a time scale which is dynamic compared with both $\mu \mathrm{SR}$ and NMR, this elastic energy scale remains $\sim \frac{1}{2} \%$ or less of the full spin excitation bandwidth of these systems. We henceforth refer to the corresponding magnetism elucidated by neutron scattering as static or quasistatic, but this proviso should be kept in mind, especially when comparing between different techniques.

Nd-LSCO has played a special role within this field, as Nd-LSCO $x=0.125$ was the first cuprate in which both parallel static spin stripe order and charge stripe order were observed [36]. Charge stripe order, with an incommensurate ordering wave vector twice that of the spin stripe order, is a natural consequence of the stripe picture, as it is the charge stripes that introduce a $\pi$ phase shift into the local $\pi-\pi$ antiferromagnetism. At the $x=0.125$ doping level, superconductivity is suppressed in Nd-LSCO to very low temperatures, with $T_{c} \sim 3 \mathrm{~K}[37,38]$. This phenomenon is known as the " $\frac{1}{8}$ anomaly." Nd-LSCO is also of interest as its maximum superconducting $T_{c}$ is relatively low, $\sim 15 \mathrm{~K}$ [39], compared with either LSCO, $\sim 40 \mathrm{~K}$ [40], or LBCO, $35 \mathrm{~K}$ [32]. This makes it easier to quench superconductivity with practical magnetic field strengths in Nd-LSCO and to thereby explore the normal state properties at doping levels that would give rise to superconductivity at zero field.

Such a deep suppression in superconducting $T_{c}$ also occurs in LBCO, and coexisting static, parallel spin and charge stripes were later observed in this system around $x=0.125$, with static, parallel spin stripes observed to $x \sim 0.135$ [32]. In contrast, LSCO displays a much milder suppression of $T_{c}$ at $x=0.125$. Both static parallel spin stripes and parallel charge stripes have been observed in LSCO, with static spin stripes observed to $x=0.15$ [41] and parallel charge stripes observed in the vicinity of $x=0.125$ [42]. A very recent study suggests that charge-density waves (CDWs) can be observed at doping levels as high as $x=0.21$ [43]. The combination of the relatively high onset temperature for charge stripes and an only modestly suppressed superconducting $T_{c}$ at the $\frac{1}{8}$ anomaly in LSCO has allowed the interaction between parallel charge stripes and superconductivity to be well studied. The $\mathrm{x}$ ray scattering intensity associated with the parallel charge stripes in LSCO around $x=0.125$ clearly diminishes on entering the superconducting state, providing compelling evidence for competition between superconductivity and charge stripes [42].

This body of work shows clearly that, while $x=0.05$ is a well-defined minimum doping level for the appearance of static, parallel spin stripes in each of Nd-LSCO, LBCO, and LSCO, the extent of this parallel spin stripe phase and how it ends is much less clear. Various phase diagrams for singlelayer, hole-doped cuprates show the static, parallel spin stripe phase to fade away by $x \sim 0.135$ for LBCO [32] and $x \sim 0.15$ for Nd-LSCO, with more limited data to $x \sim 0.2$ [37].

In this paper, we present elastic and inelastic neutron scattering measurements on four single crystals of Nd-LSCO with $x=0.125,0.19,0.24$, and 0.26 . The $x=0.26$ doping level is close to the end of superconductivity in Nd-LSCO [44], while the $x=0.24$ sample is close to the proposed quantum critical point, $p^{*}=0.23 \pm 0.005$, associated with the pseudogap phase in Nd-LSCO [45]. These measurements show welldefined, but non-resolution-limited magnetic Bragg peaks to appear at low temperatures in all of these samples, significantly extending the range of concentrations over which static, parallel spin stripes have been observed in Nd-LSCO and challenging the notion that static magnetism ends at or before $p^{*}$ [39]. We report that the effective $\mathrm{Cu}$ ordered moment within the spin stripe structure at low temperatures drops off monotonically and steeply from $x=0.125$ to higher dopings, with a concomitant decrease in the in-plane correlation length. This provides a reasonable explanation for why this signal has been so difficult to observe until now. On the basis of these measurements we propose a phase diagram for parallel spin stripes in Nd-LSCO that uncouples the connection between static magnetism and the pseudogap phase. Instead we show that the static magnetic order spans the entirety of the superconducting dome, $0.05 \leqslant x \leqslant 0.26$. Consequently, the superconducting state at any $x$ in $\mathrm{Nd}-\mathrm{LSCO}$ is entered from a state with extensive static, parallel spin stripe order. We conclude that this parallel spin stripe state, in contrast with the parallel charge stripe state, does not compete with superconductivity in Nd-LSCO, but is a prerequisite for it.

\section{METHODS}

Crystal growth. High-quality single crystals of Nd-LSCO with $x=0.125,0.19,0.24$, and 0.26 were grown using the traveling solvent floating zone technique at McMaster University with each resulting single-crystal sample weighing between 3.5 and $5 \mathrm{~g}$. The single crystals were produced using a four-mirror Crystal Systems halogen lamp image furnace at approximate growth speeds of $0.68 \mathrm{~mm} / \mathrm{h}$, and growths lasting for approximately 1 week each. The $\mathrm{Sr}$ concentration of each single crystal was determined by careful correlation of the structural phase transition temperatures with preexisting 
phase diagrams, as described in Ref. [44]. Further details regarding the material preparation and single-crystal growth of these samples, as well as determination of their stoichiometry, are reported in Ref. [44]. The Nd-LSCO sample with $x=0.19$ was composed of two coaligned single crystals. All single crystals were scanned using a backscattering Laue instrument to assess their single-crystal nature. Neutron diffraction measurements showed mosaic spreads of less than $0.5^{\circ}$ in all crystals, attesting to their high-quality, single crystallinity.

Triple-axis neutron scattering experiment. Nd-LSCO single crystals with $x=0.19,0.26$ were studied using the triple-axis neutron spectrometer (TAS) Taipan at the Australian Centre for Neutron Scattering (ACNS), Australian Nuclear Science and Technology Organisation (ANSTO), while the TAS experiment for the $x=0.24$ sample was conducted using the HB3 TAS instrument at the High Flux Isotope Reactor (HFIR) at Oak Ridge National Laboratory. Experiments using both Taipan and HB3 employed pyrolytic graphite monochromators, analyzers, and filters and employed the same fixed final neutron energies: $E_{f}=14.7 \mathrm{meV}$. Horizontal beam collimations of open- $40^{\prime}-40^{\prime}$-open for Taipan and $48^{\prime}-40^{\prime}-40^{\prime}-120^{\prime}$ for HB3 were used. The resulting energy resolution of the two sets of measurements was therefore similar, $\sim 0.9 \mathrm{meV}$. For all TAS measurements the crystals were loaded in pumped ${ }^{4} \mathrm{He}$ cryostats with a base temperature of $1.5 \mathrm{~K}$.

Time-of-flight neutron scattering experiment. Time-offlight (TOF) neutron chopper spectrometer measurements were also carried out on the $x=0.125,0.19$, and 0.24 single crystals using the direct geometry spectrometer SEQUOIA at the Spallation Neutron Source, Oak Ridge National Laboratory [46]. These measurements were performed using $E_{i}=60 \mathrm{meV}$ neutrons, which gave an energy resolution of $\sim 1.2 \mathrm{meV}$ at the elastic position. For all TOF measurements, the crystals were loaded in closed-cycle refrigerators with a base temperature of $5 \mathrm{~K}$.

\section{RESULTS}

\section{A. Elastic neutron scattering}

Elastic neutron scattering scans of the form $(H, 1 / 2,0)$ and $(1 / 2, K, 0)$ (using tetragonal notation where the $a, b$ axes are $\sim 3.76 \AA$ at room temperature) were carried out for the Nd-LSCO single-crystal samples with $x=0.19,0.24$, and 0.26 with a base temperature $T=1.5 \mathrm{~K}$. These elastic scattering data are shown in Fig. 1. Four IC $\operatorname{AF}\left(\frac{1}{2} \pm \delta, \frac{1}{2}, 0\right)$ and $\left(\frac{1}{2}, \frac{1}{2} \pm \delta, 0\right)$ quasi-Bragg peaks are observed, as expected for twinned orthorhombic structures. Schematic trajectories of these scans in reciprocal space are shown in the insets of Figs. 1(a) and 1(b). All the IC AF scattering is non-resolutionlimited in $\boldsymbol{Q}$ space, which is why we refer to these sharp diffraction features as quasi-Bragg peaks.

These results show that static IC AF order exists at $T=$ $1.5 \mathrm{~K}$ in all three single crystals: one at optimal doping and below $p^{*}$, and two above $p^{*}$. The intensity for the $x=0.19$ sample is considerably stronger than that of $x=0.24$ or $x=$ 0.26 , but the signals are otherwise qualitatively similar to each other. They are also qualitatively similar to earlier elastic neutron scattering results on an $x=0.125$ sample by Tranquada

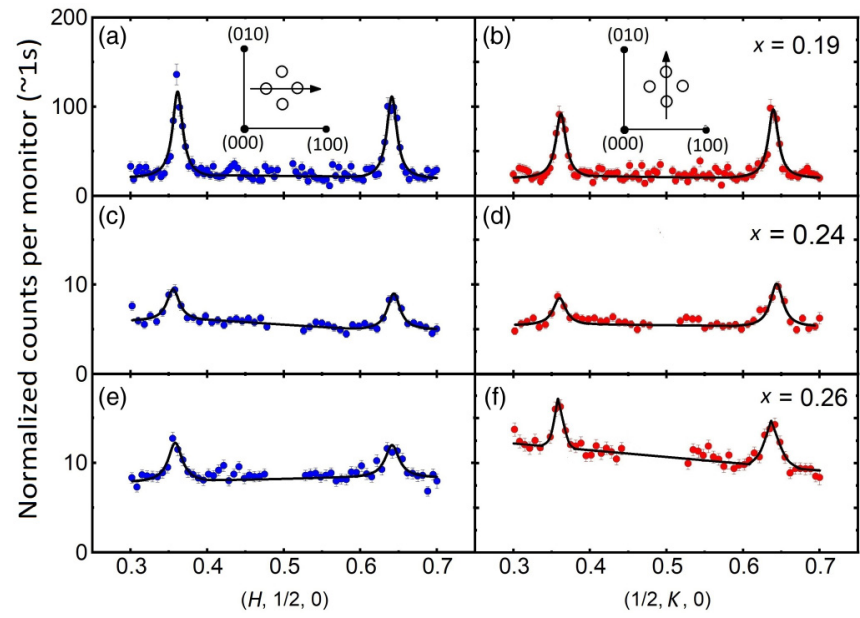

FIG. 1. Elastic TAS neutron scattering scans in reciprocal space for (a) and (b) $x=0.19$, (c) and (d) $x=0.24$, and (e) and (f) $x=$ 0.26 single crystals of Nd-LSCO at $T=1.5 \mathrm{~K}$. The IC peaks can be observed in all three samples at reciprocal space positions $\left(\frac{1}{2} \pm \delta, \frac{1}{2}, 0\right)$ and $\left(\frac{1}{2}, \frac{1}{2} \pm \delta, 0\right), \delta \approx 0.14$. The insets in (a) and (b) illustrate the $H$ and $K$ scans employed in reciprocal space. The vertical axis displays neutron intensity, counts per monitor for approximately $1 \mathrm{~s}$. The black lines going through the data points are fits to the data described by Eq. (1). Data have been normalized to $(1,1,0)$ Bragg peak intensities to account for differences in effective volume between the three samples.

and co-workers $[36,37,47,48]$. We therefore conclude that all Nd-LSCO single-crystal samples with $0.125 \leqslant x \leqslant 0.26$ display quasi-Bragg peaks at $T=1.5 \mathrm{~K}$, which corresponds to IC AF order that is well defined in $Q$ and static on the time scale of the neutron scattering measurements $\left(\sim 10^{-10} \mathrm{~s}\right)$.

The order parameters, or the temperature dependence of the Bragg intensities, were measured at the IC AF position $(0.5,0.64,0)$, which is $\left(\frac{1}{2}, \frac{1}{2}+\delta, 0\right)$ with $\delta=0.14$, with careful thermal equilibration of the samples. These order parameter measurements were performed for the Nd-LSCO single crystals with $x=0.19,0.24$, and 0.26 , as shown in Figs. 2(a)-2(c), respectively. All three samples display a pronounced upturn in their intensity below $\sim 4 \mathrm{~K}$, which is marked with a vertical fiducial in Fig. 2. Similar behavior was also observed in the corresponding order parameter measurement of Nd-LSCO with $x=0.125$ by Tranquada et al. [37]. There, this upturn was successfully modeled as arising from the coupling between $\mathrm{Cu}^{2+}$ and $\mathrm{Nd}^{3+}$ moments and the onset of $\mathrm{Nd}^{3+}$ magnetic correlations at low temperatures.

This $\mathrm{Cu}^{2+}-\mathrm{Nd}^{3+}$ coupling leads to the development of pronounced $3 \mathrm{D}$ correlations which peak at ordering wave vectors $\left(\frac{1}{2} \pm \delta, \frac{1}{2}, 0\right)$ and $\left(\frac{1}{2}, \frac{1}{2} \pm \delta, 0\right)$, which is with $L=0$ [48]. At temperatures above $\sim 4 \mathrm{~K}$, the IC AF Bragg scattering takes on a progressively more $2 \mathrm{D}$ nature, with scattering extended along $L$ in reciprocal space [48]. The $3 \mathrm{D}$ to $2 \mathrm{D}$ nature of the IC AF elastic scattering from low to high temperature has been previously discussed for Nd-LSCO with $x=0.125$ in Ref. [48]. It has also been studied in other 214 cuprates such as LBCO in Ref. [49], for example, where time-of-flight neutron data differentiate between 3D IC antiferromagnetism 


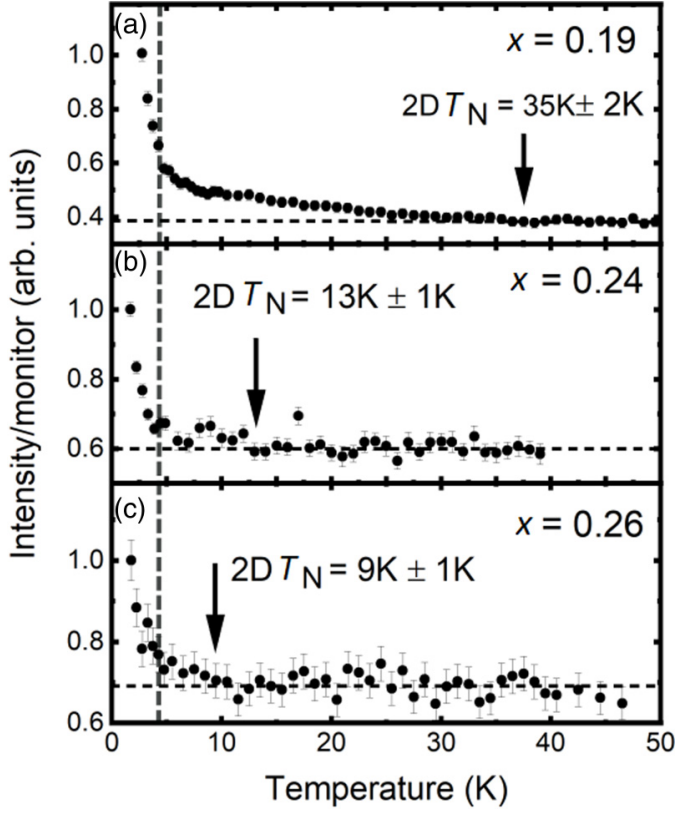

FIG. 2. Elastic temperature scans on one of the four IC AF peaks at $(0.5,0.64,0)$. The data were taken upon warming the samples from base temperature $\sim 1.5 \mathrm{~K}$ up to 50,40 , and $46 \mathrm{~K}$ with careful equilibration for each of the $x=0.19,0.24$, and 0.26 samples, in (a), (b), and (c), respectively. The vertical dashed line indicates $T=4 \mathrm{~K}$, below which a pronounced upturn in all intensities is observed, due to $\mathrm{Nd}^{3+}-\mathrm{Cu}^{2+}$ coupling. Horizontal dashed lines mark the high-temperature background, and the arrows indicate where the 2D IC AF Bragg scattering signal departs from the background in each sample. The estimated 2D $T_{N}$ for $x=0.19$ is $35 \pm 2 \mathrm{~K}$ (a), for $x=0.24$ it is $13 \pm 1 \mathrm{~K}$ (b), and for $x=0.26$ it is $9 \pm 1 \mathrm{~K}$ (c). The intensity at $T=1.5 \mathrm{~K}$ has been normalized to 1 .

appearing as a Bragg "spot" and 2D magnetic magnetic scattering extending into rods of scattering perpendicular to the $\mathrm{CuO}_{2}$ planes.

In addition, the $\mathrm{Cu}^{2+}-\mathrm{Nd}^{3+}$ coupling induces a rotation of the $S=1 / 2 \mathrm{Cu}^{2+}$ moments out of the basal plane, so as to be more parallel with the $\mathrm{Nd}^{3+}$ moments, which themselves are largely constrained to be along $c$ due to crystal field effects. As $c$ is perpendicular to the $\left(\frac{1}{2} \pm \delta, \frac{1}{2}, 0\right)$ and $\left(\frac{1}{2}, \frac{1}{2} \pm \delta, 0\right)$ ordering wave vectors, the intensity of the magnetic Bragg scattering also increases as a consequence of the $\mathrm{Cu}^{2+}$ moments being more perpendicular to $\boldsymbol{Q}$, which results in higher magnetic Bragg intensities due to the polarization dependence of the magnetic neutron scattering cross section.

As the concentration of $\mathrm{Nd}$ is the same across this $\mathrm{Nd}$ LSCO series, the same phenomenology is expected at all $x$. As can be seen from Fig. 2, this is indeed the case with a pronounced factor of $\sim 4$ enhancement in the strength of the IC AF Bragg scattering at $\left(\frac{1}{2} \pm \delta, \frac{1}{2}, 0\right)$ and $\left(\frac{1}{2}, \frac{1}{2} \pm \delta, 0\right)$ from $T \sim 4 \mathrm{~K}$ to $T \sim 1.5 \mathrm{~K}$ for all $x$. Of course, such an enhancement would not occur in the absence of $\mathrm{Nd}$; hence the identification of the IC AF Bragg scattering at low temperatures is considerably easier in Nd-LSCO, as compared with LBCO or LSCO, especially at high doping.

The order parameter measurements also allow us to estimate the onset of the 2D IC antiferromagnetism, 2D $T_{N}$.

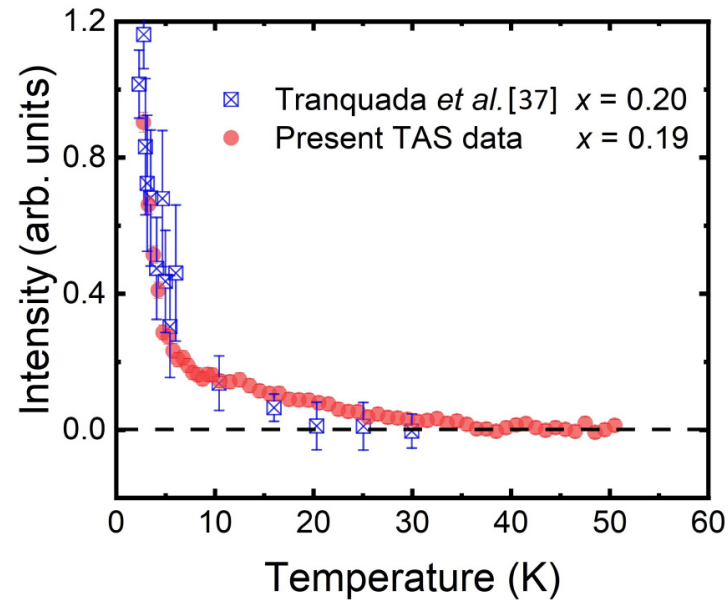

FIG. 3. Comparison of the present TAS data order parameter on the $x=0.19$ sample and that reported earlier for an $x=0.20$ sample [37]. The two elastic scattering data sets show very good agreement with each other. However, the present data on the $x=0.19$ sample have much smaller error bars (smaller than the data points) and much higher temperature point density, aiding in a more accurate estimate of $2 \mathrm{D} \mathrm{T} \mathrm{T}_{N}=35 \pm 2 \mathrm{~K}$.

From earlier work by Tranquada et al. [37] on Nd-LSCO at $x=0.125$ and $0.15,2 \mathrm{D} T_{N}$ is known to peak at $\sim 50 \mathrm{~K}$ for $x=0.125$, where IC charge order is strongest, and the superconducting $T_{c}$ is a local minimum, $\sim 3 \mathrm{~K}$. Our order parameter data in Fig. 2 show $2 \mathrm{D} T_{N}$ to correspond to where the 2D IC AF Bragg scattering departs from a high-temperature background. The background employed is $40<T<50 \mathrm{~K}$ for $x=0.19,23<T<40 \mathrm{~K}$ for $x=0.24$, and $20<T<46 \mathrm{~K}$ for $x=0.26$. This gives estimates for $2 \mathrm{D} T_{N}$ of $35 \pm 2 \mathrm{~K}$ for $x=0.19 ; 13 \pm 1 \mathrm{~K}$ for $x=0.24$; and $9 \pm 1 \mathrm{~K}$ for $x=0.26$, as marked by the arrows in Figs. 2(a)-2(c), respectively.

The same earlier work by Tranquada et al. [37] on $\mathrm{Nd}$ LSCO also measured the order parameter for an $x=0.2$ sample, very close in composition to our $x=0.19$ single crystal. Figure 3 shows these two data sets overlaid on each other, and the raw data are clearly consistent with each other. However, the error bars associated with the neutron intensity and the temperature point density associated with the $x=$ 0.19 data are much improved over the preexisting $x=0.2$ data, allowing us a better estimate of $2 \mathrm{D} T_{N}=35 \pm 2 \mathrm{~K}$. The earlier estimate of 2D $T_{N} \sim 20 \mathrm{~K}$ had been employed to extrapolate the end of static magnetism in Nd-LSCO at $p^{*}$ [15]. We employ the 2D $T_{N} \sim 35 \pm 2 \mathrm{~K}$ value for $x=0.19$ in all subsequent discussions.

TOF elastic neutron scattering data, complementary to the TAS data shown in Fig. 1, are displayed within the $(H, K,-4<L<4)$ plane of reciprocal space, for $x=0.125$, 0.19 , and 0.24 single crystals in Fig. 4 . The $x=0.19$ and 0.24 single-crystal samples are the same single-crystal samples used for the TAS measurements in Fig. 1. All three data sets show a difference between $T=5 \mathrm{~K}$ data and those taken at a temperature equal to or above 2D $T_{N}(T=60 \mathrm{~K}$ for $x=0.125$ and $T=35 \mathrm{~K}$ for each of $x=0.19$ and 0.24$)$. These data were extracted from four-dimensional (three $\boldsymbol{Q}$ dimensions, as well as energy) data sets taken with the TOF chopper spectrometer 


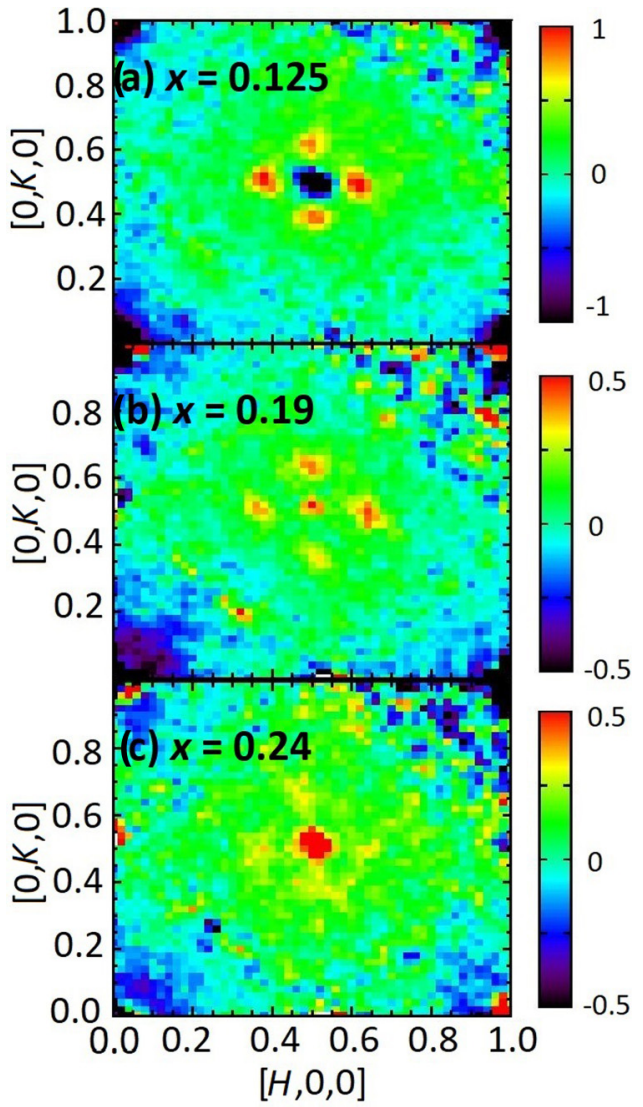

FIG. 4. Elastic time-of-flight (TOF) neutron data from $\mathrm{Nd}$ LSCO with (a) $x=0.125$, (b) $x=0.19$, and (c) $x=0.24$ in the $[H, K,-4 \leqslant L \leqslant 4]$ plane; that is with an integration in $L$ from -4 to 4 reciprocal lattice unit (r.l.u.). These results show the subtraction of a high-temperature data set from a low-temperature data set, $5 \mathrm{~K}$ $-60 \mathrm{~K}, 5 \mathrm{~K}-35 \mathrm{~K}$, and $5 \mathrm{~K}-35 \mathrm{~K}$, respectively. The IC AF magnetic peaks around $[1 / 2,1 / 2,0]$, arising from $2 \mathrm{D}$ parallel stripe order, are sharpest and most intense for $x=0.125$ in (a) and gradually broaden and weaken with higher doping, 0.19 in (b) and 0.24 in (c). The data have been normalized to a combination of incoherent elastic scattering and that from crystal field excitations of $\mathrm{Nd}^{3+}$ for all samples.

SEQUOIA. The elastic scattering data are integrated in energy over the range $-2<E<2 \mathrm{meV}$, which encompasses the $\sim 1$. $-\mathrm{meV}$ energy resolution for SEQUOIA measurements employing $E_{i}=60 \mathrm{meV}$. The integration over $-4<L<4$ is necessary to capture sufficient 2D IC AF Bragg intensity, which is extended along $L$ at $T=5 \mathrm{~K}$ and concentrated at relatively low $\boldsymbol{Q}$, due to the magnetic form factor of $\mathrm{Cu}^{2+}$. Allowed nuclear Bragg peaks occur in these orthorhombic structures at wave vectors such as $\left(\frac{1}{2}, \frac{1}{2}, L= \pm 1, \pm 2, \pm 3\right)$, using tetragonal notation. Hence relatively strong nuclear intensity is observed at these positions. These structural zone centers also have low-energy acoustic phonons associated with them, whose intensity increases with increasing temperature, and which, while weak, can be picked up within the energy integration performed here. As a result, the temperature difference plots shown in Fig. 4 have relatively strong positive or negative intensity at the $\left(\frac{1}{2}, \frac{1}{2},-4<L<4\right)$ position, due to the subtraction of two relatively large nuclear intensities. This is an artifact of the subtraction between data sets at two different temperatures.

These measurements were performed on three single crystals of similar size and under very similar conditions. Although a normalization of the strength of the IC AF Bragg intensities is straightforward, and will be carried out below, it is useful to consider the data directly. We can see that the IC AF Bragg intensity at $T=5 \mathrm{~K}$ is well defined and relatively strong for $x=0.125$, becoming substantially weaker for $x=$ 0.19 , and then both weak and broad for $x=0.24$.

An analysis of the elastic TAS data in Fig. 1, and the elastic TOF data in Fig. 4, was carried out so as to quantitatively estimate the relative strength of the IC AF Bragg scattering as a function of $x$ for our $x=0.125,0.19,0.24$, and 0.26 samples at both $T=1.5$ and $5 \mathrm{~K}$. The $H$ and $K$, elastic TAS scans in Fig. 1 were fit to Lorentzian line shapes of the form

$$
S(Q, \hbar \omega=0)=\frac{I_{0}}{\left(Q-Q_{0}\right)^{2}+\left(\frac{1}{2} \Gamma\right)^{2}}
$$

(where $\Gamma$ indicates the full width at half maximum of the function and $Q_{0}$ is its center) with the purpose of extracting both the integrated intensity of the IC AF Bragg scattering and its in-plane correlation length. The intensity of the IC AF Bragg scattering was normalized against rocking curve measurements of the intensity of structurally allowed $(1,1,0)$ Bragg peaks, such that the intensities of the IC AF Bragg scattering at $T=1.5 \mathrm{~K}$ could be quantitatively compared between the $x=0.19,0.24$, and 0.26 samples.

A similar normalization protocol was followed for the IC AF Bragg scattering in the $x=0.125,0.19$, and 0.24 samples at $T=5 \mathrm{~K}$, using the TOF data in Fig. 4. As these elastic scattering data are part of much larger inelastic scattering data sets, we can normalize the IC AF integrated Bragg scattering to that of crystalline electric field (CEF) excitations near $\sim 12$ and $24 \mathrm{meV}$. A study on these CEF excitations will be reported separately, but their intensity depends primarily on the amount of $\mathrm{Nd}$ present in the samples, which is the same in all cases.

These results are summarized in Fig. 5(a) for the integrated intensities and Fig. 5(b) for the correlation lengths. In both cases our results are put into the context of previous measurements on Nd-LSCO single-crystal samples [37,47]. The primary conclusions are that the $x$ dependence of the intensity of the IC AF Bragg peaks, which goes as the square of the sublattice magnetization, $\langle M\rangle^{2}$, is consistent between our data at $T=1.5 \mathrm{~K}$ on $x=0,19,0.24$, and 0.26 samples and those of Tranquada et al. on $x=0.125,0.15$, and 0.20 samples [37]. At this low temperature, $T=1.5 \mathrm{~K}$, the influence of the $\mathrm{Cu}^{2+}-\mathrm{Nd}^{3+}$ coupling is profound, and the scattering has significant 3D correlations. In contrast, our TOF data for $x=$ $0.125,0.19$, and 0.24 samples at $T=5 \mathrm{~K}$ are much more $2 \mathrm{D}$ in nature. We can see that this TOF integrated static magnetic intensity also falls off sharply with $x$ from $x=0.125$ to 0.24 , but at about half the rate seen for the TAS results at $T=1.5 \mathrm{~K}$. We attribute this difference between the $x$ dependence of the static magnetism at $T=1.5 \mathrm{~K}$ and that at $T=5 \mathrm{~K}$, to an $x$ dependence in the nature of the effectiveness of the coupling between $\mathrm{Cu}^{2+}$ and $\mathrm{Nd}^{3+}$ magnetic moments. We conclude that the coupling is stronger and thus more efficient for smaller $x$, where more $\mathrm{Cu}^{2+}$ moment is present. 


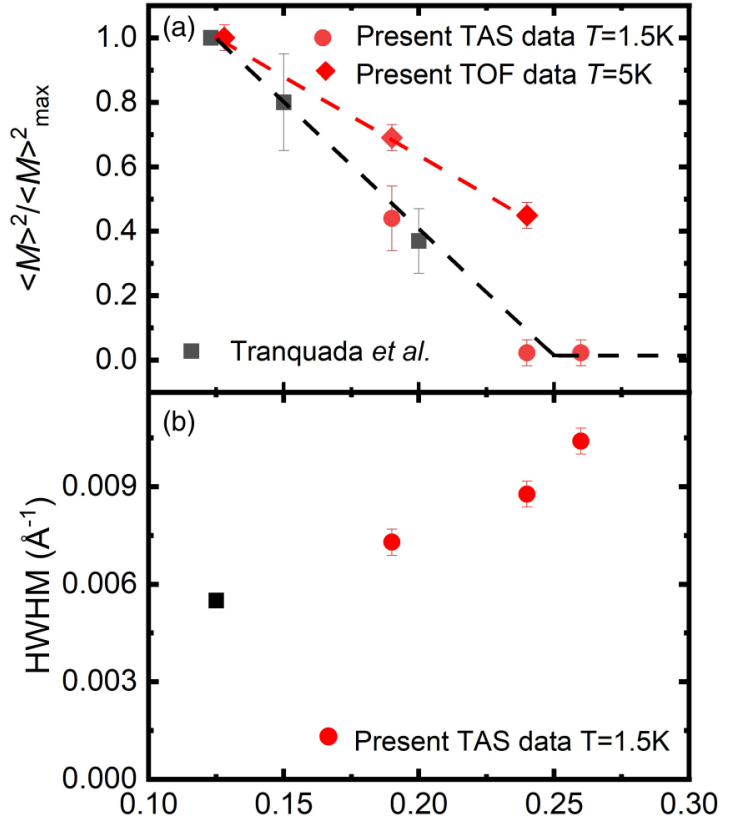

FIG. 5. (a) The square of the sublattice magnetization at 1.5 and $5 \mathrm{~K}$, normalized to $x=0.12$. The black squares represent preexisting data from Tranquada et al. [37]. The red circles represent the current TAS data, which show consistency with the preexisting data. The red diamonds represent the $5-\mathrm{K}$ data taken as the integrated intensities of the IC AF peaks in Fig. 4. Data have been normalized to a combination of incoherent Bragg scattering and crystal field excitation of $\mathrm{Nd}^{3+}$ for all samples. (b) The inverse magnetic correlation lengths, which are taken as half-width at half maximum (HWHM), of the current IC AF peaks in $x=0.19,0.24$, and 0.26 are shown, along with preexisting $x=0.12$ (black square) data from Tranquada et al. [47].

Earlier work by Tranquada et al. on $x=0.125$ concluded that the ordered $\mathrm{Cu}^{2+}$ moment at low temperatures was $0.1 \pm$ $0.03 \mu_{B}$ [48]. Our work then suggests that the ordered $\mathrm{Cu}^{2+}$ moment participating in the $2 \mathrm{D}$ IC AF structure at $T=5 \mathrm{~K}$ in our $x=0.24$ single-crystal samples is roughly $40 \%$ smaller, thus $\sim 0.06 \mu_{B}$. For context, the ordered $\mathrm{Cu}^{2+}$ moment in the ordered state of $\mathrm{La}_{2} \mathrm{CuO}_{4}$, as determined by neutron diffraction, is $0.6 \pm 0.05 \mu_{B}$ [50], while that for $\mathrm{Nd}_{2} \mathrm{CuO}_{4}$ is $0.46 \pm 0.05 \mu_{B}[50]$.

The in-plane magnetic correlation lengths at $T=1.5 \mathrm{~K}$ are plotted in Fig. 5(b) for our single crystals $x=0.19,0.24$, and 0.26 . Previous studies on IC AF order for $x=0.125$ had already determined that the relevant magnetic Bragg features had finite in-plane correlation length, in spite of the fact that the IC AF order is at its strongest at this doping level [48]. Our results are consistent with those for $x=0.125$, in that the sequence of low-temperature in-plane correlation lengths is seen to decrease monotonically with doping (the half-width at half maximum increases monotonically), and the overall trend from $x=0.125$ to 0.26 is an approximately linear relationship.

\section{B. Low-energy inelastic magnetic scattering}

The low-energy spin fluctuations within the static, parallel spin stripe state of Nd-LSCO were also investigated using TAS spectroscopy. Constant- $\boldsymbol{Q}$ measurements were performed

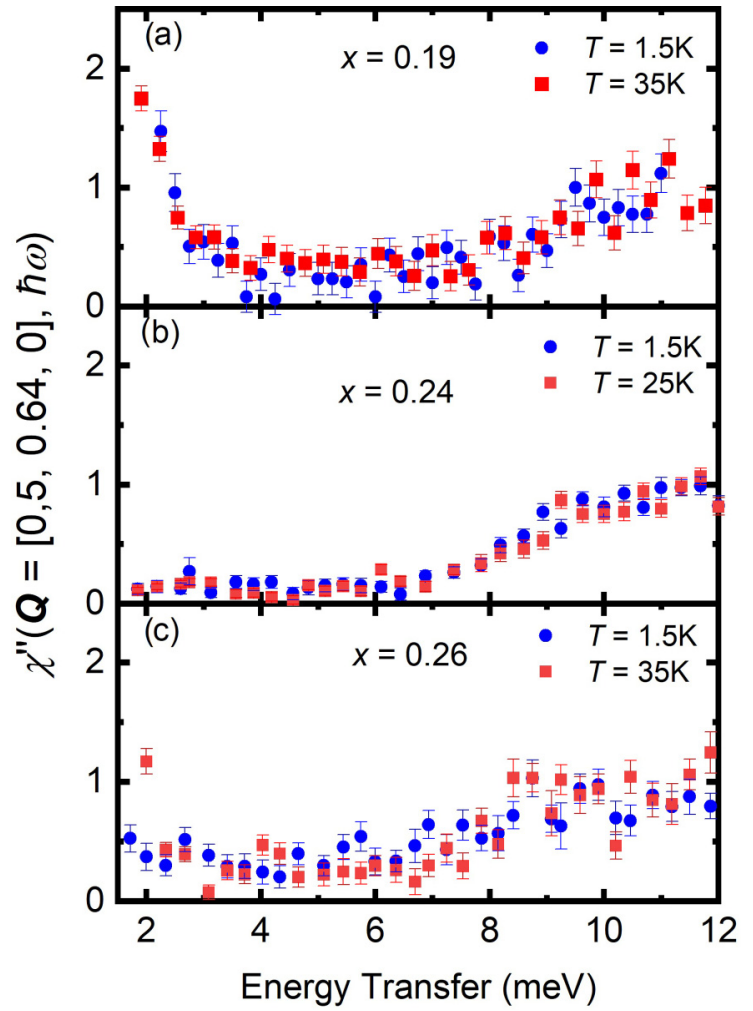

FIG. 6. Energy scans of $\chi^{\prime \prime}(Q, \hbar \omega)$ taken from TAS constant- $\boldsymbol{Q}$ measurements at $Q=[0.5,0.64,0]$ : one of the four incommensurate AF peak positions around the $[0.5,0.5,0]$ position. Data have been normalized to the intensity of the crystal field excitation from $\mathrm{Nd}^{3+}$ around $11 \mathrm{meV}$.

at the IC AF zone center, $(0.5,0.64,0)$, as a function of energy transfer, as shown for $x=0.19,0.24$, and 0.26 in Figs. 6(a) 6(c), respectively. Constant-energy scans were also performed as a function of $\boldsymbol{Q}$, across an IC AF zone center with scans of the form $(0.5, K, 0)$, as shown in Fig. 7 for the $x=0.19$ single crystal.

Figures 6-8(a) show the imaginary part of the dynamic susceptibility $\chi^{\prime \prime}(Q, \hbar \omega)$ as a function of both $\hbar \omega$ and $\boldsymbol{Q}$, respectively. This quantity is related to the inelastic neutron scattering intensity, the dynamic structure factor $\mathrm{S}(Q, \hbar \omega)$, by

$$
S(Q, \hbar \omega)=\left[1-\exp \left(\frac{-\hbar \omega}{k_{B} T}\right)\right]^{-1} \times \chi^{\prime \prime}(Q, \hbar \omega) .
$$

Isolating $\chi^{\prime \prime}(Q, \hbar \omega)$ from the dynamic structure factor $S(Q, \hbar \omega)$ involves making a robust estimate of the background and then dividing the inelastic neutron scattering signal by the Bose factor, $\left[1-\exp \left(\frac{-\hbar \omega}{k_{B} T}\right)\right]^{-1}$. This has been done for the data shown in Figs. 6-8(a), using the scattering away from IC AF Bragg positions, such as $(0.5,0.68,0)$, to estimate the background level.

Inspection of the low energy dependence of $\chi^{\prime \prime}(Q, \hbar \omega)$ at $Q=(0.5,0.64,0)$ in Fig. 6 shows that this spectral weight is qualitatively different for the optimally doped $x=0.19$ sample, with superconducting $T_{c} \sim 14 \mathrm{~K}$, as compared with either of the $x=0.24$ or 0.26 single crystals which lie beyond the pseudogap quantum critical point $p^{*}$ and close to the end 


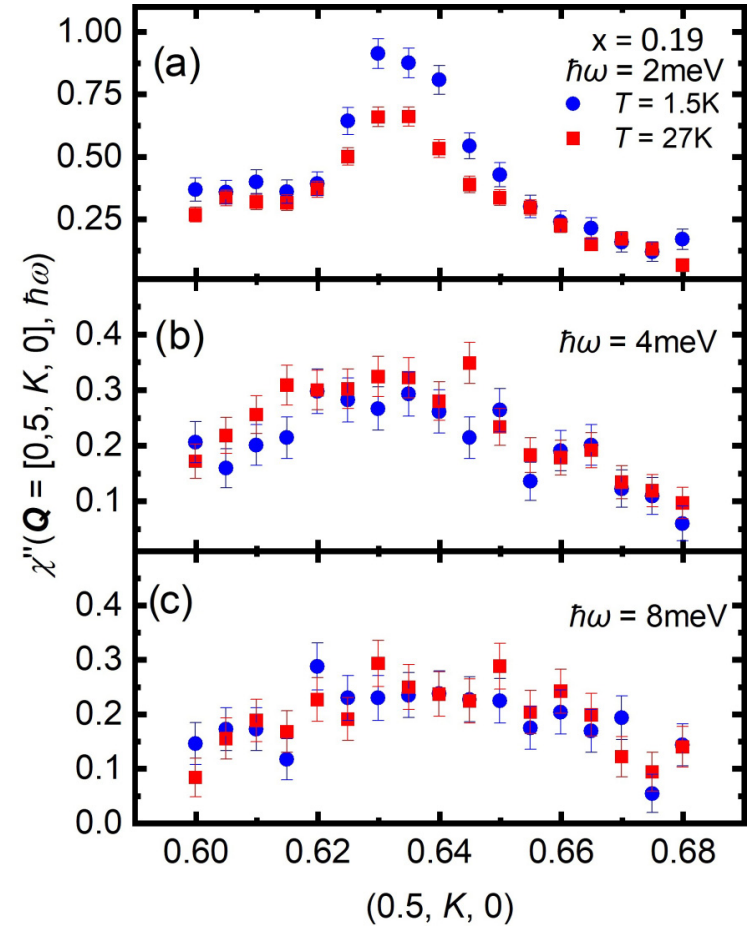

FIG. 7. $\chi^{\prime \prime}(Q, \hbar \omega)$ for $x=0.19$ is shown for $Q=[0.5,0.64,0]$ and $\hbar \omega=2,4$, and $8 \mathrm{meV}$ in (a), (b), and (c), respectively, from TAS constant-energy-transfer $(\hbar \omega)$ scans at both low $(T=1.5 \mathrm{~K})$ and high temperatures $(T=27.5 \mathrm{~K})$.

of the superconducting dome. While all three samples show pronounced inelastic scattering which peaks near a low-lying CEF excitation at $\sim \hbar \omega=11 \mathrm{meV}$, only the $x=0.19$ sample shows strong quasielastic scattering which rises up in intensity below $\sim 4 \mathrm{meV}$. For the higher-doped samples, $x=0.24$ and 0.26 , very little inelastic magnetic spectral weight is obvious at energies less than $\sim 4 \mathrm{meV}$ in these measurements.

More detailed constant-energy $\boldsymbol{Q}$ scans show that this low-energy spectral weight is strongly peaked at the IC AF ordering wave vector, as can be seen in Fig. 7 for $x=0.19$. A strong peak is seen in the $\chi^{\prime \prime}(Q, \hbar \omega=2 \mathrm{meV})$ data that is not seen in the corresponding data at higher energies, $\hbar \omega=4$ or $8 \mathrm{meV}$. At $\hbar \omega=2 \mathrm{meV}$, the strength of $\chi^{\prime \prime}(Q, \hbar \omega)$ decreases by $\sim 40 \%$ on warming from $T=1.5 \mathrm{~K}$ to $T=27 \mathrm{~K}$. $\chi^{\prime \prime}(Q, \hbar \omega)$ at $\hbar \omega=4$ or $8 \mathrm{meV}$ remains peaked around the IC AF ordering wave vector, but it is both considerably broader in $Q$ and weaker in intensity than that at $2 \mathrm{meV}$. As expected, $\chi^{\prime \prime}(Q, \hbar \omega)$ at $\hbar \omega=4$ or $8 \mathrm{meV}$ shows little or no temperature dependence to $27 \mathrm{~K}$.

We have measured the temperature dependence of the IC AF inelastic spectral weight at $\hbar \omega=2 \mathrm{meV}$ for the $x$ $=0.19$ sample. This is shown in Fig. 8(a), where $\chi^{\prime \prime}[Q=$ $(0.5,0.64,0), \hbar \omega=2 \mathrm{meV}]$ is shown as a function of temperature from $T=1.5 \mathrm{~K}$ to $T=30 \mathrm{~K}$. This temperature dependence is the low-energy, inelastic equivalent of the order parameter, which is the elastic scattering measured at the same IC AF wave vector shown in Figs. 1 and 2. The order parameter for $x=0.19$, Fig. 2(a), shows that the 2D $T_{N}=35 \pm 2 \mathrm{~K}$. Furthermore, it rises sharply with decreasing temperature at low temperatures, due to coupling between the

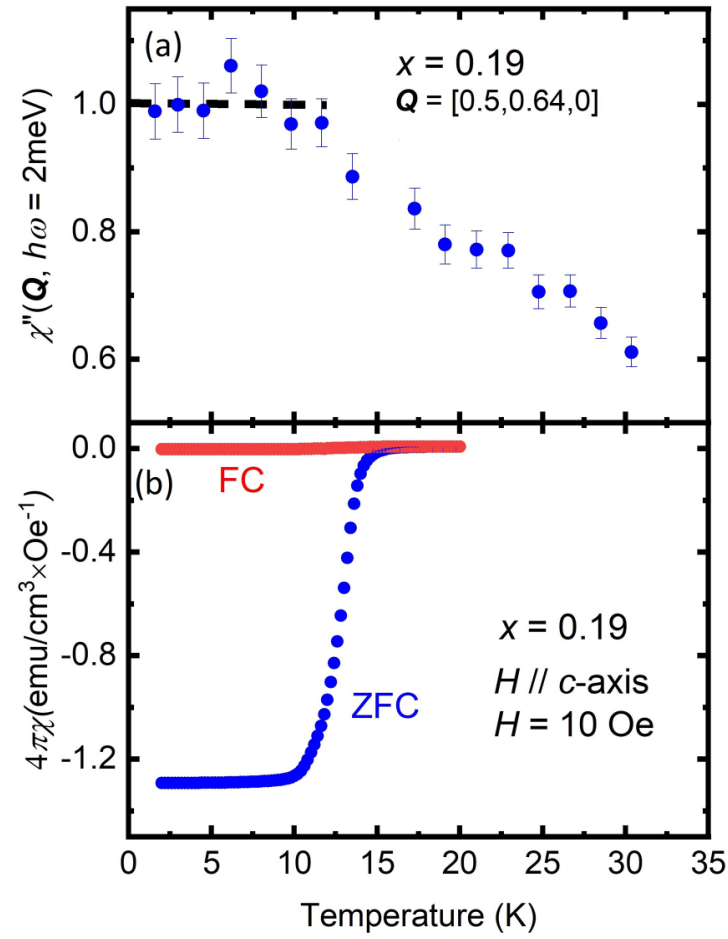

FIG. 8. (a) The temperature dependence of the low-energy spectral weight of the parallel stripe fluctuations, $\chi^{\prime \prime}(Q, \hbar \omega)$, as a function of temperature for $Q=[0.5,0.64,0]$ and $\hbar \omega=2 \mathrm{meV}$ is shown for Nd-LSCO with $x=0.19$. These data were derived from TAS measurements. The horizontal dashed line indicates the plateau behavior for $T \leqslant T_{c} \sim 13.5 \mathrm{~K}$. (b) Field-cooled (FC) and zero-field-cooled (ZFC) magnetic susceptibility measurements on a small single crystal of the $x=0.19 \mathrm{Nd}$-LSCO sample with a magnetic field of $10 \mathrm{Oe}$ applied parallel to the $c$ axis show $\mathrm{T}_{c} \sim 13.5 \mathrm{~K}$.

$\mathrm{Cu}^{2+}$ and $\mathrm{Nd}^{3+}$ moments which induces substantial 3D spin correlations [48]. However, the spin fluctuations captured in $\chi^{\prime \prime}(Q, \hbar \omega=2 \mathrm{meV})$ at the IC AF wave vector rise linearly with decreasing temperature from $30 \mathrm{~K}$ to $\sim 13 \mathrm{~K}$, before leveling off at low temperatures.

Figure 8 is very interesting as it shows the spectral weight of these low-energy, parallel spin stripe fluctuations in this optimally hole-doped cuprate to saturate at temperatures below the approximate superconducting $T_{c}$ of this single crystal. In this $x=0.19$ sample, the temperatures of relevance for $2 \mathrm{D}$ magnetic order $\left(2 \mathrm{D} T_{N} \sim 35 \mathrm{~K}\right)$ and strong 3D correlations $(\sim 4 \mathrm{~K})$ are well separated from that associated with superconductivity. For ease of reference, the measured magnetic susceptibility of a small piece of the single crystal used in neutron scattering experiments is plotted in Fig. 8(b) directly below the $\chi^{\prime \prime}(Q, \hbar \omega)$ temperature dependence data for $\hbar \omega=$ $2 \mathrm{meV}$. A strong diamagnetic signal is observed with an onset near $T_{c} \sim 14.5 \mathrm{~K}$, but the midpoint on the zero-field-cooled diamagnetic susceptibility curve occurs at $\sim 13.5 \mathrm{~K}$, coincident with the start of the plateau in the inelastic, $\hbar \omega=2 \mathrm{meV}$ spectral weight at low temperatures. A similar trend in the temperature dependence of $\chi^{\prime \prime}(Q, \hbar \omega)$ at low energies has been reported for Nd-LSCO with $x=0.15$ [47], but with a lower temperature point density, making it hard to identify characteristic temperatures. 


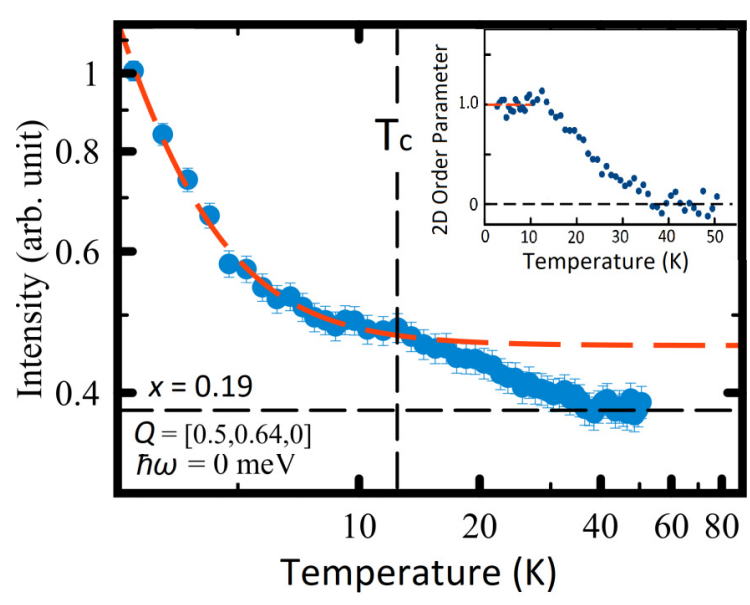

FIG. 9. The order parameter for Nd-LSCO $x=0.19$ is replotted; this is the same data as in Fig. 2(a), but on a log-log scale. An inflection point in the temperature evolution of the order parameter is clearly observed at superconducting $T_{c} \sim 13.5 \mathrm{~K}$ (indicated by the vertical dashed fiducial). The inset shows the $2 \mathrm{D}$ order parameter (2DOP) for Nd-LSCO $x=0.19$ derived from the order parameter (OP) measurements shown in the main figure and in Fig. 2(a). $2 \mathrm{DOP}(T)$ is obtained from the relation $\mathrm{OP}(T)=A(T) \times 2 \mathrm{DOP}(T)$. The amplification factor $A(T)$ is derived from fitting the $\operatorname{OP}(T)$ at $T \leqslant 12 \mathrm{~K}$, as shown with the red line in the inset. Like the lowenergy spectral weight of the parallel stripe fluctuations, $\chi^{\prime \prime}(Q=$ $[0.5,0.64,0], \hbar \omega=2 \mathrm{meV}$ ) as a function of temperature in Fig. 8, 2DOP displays the onset of its low-temperature plateau coincident with $T_{c}$.

Examination of the elastic order parameter for $x=0.19$ shows evidence for an inflection point at $T_{c} \sim 13.5 \mathrm{~K}$. Figure 9 reproduces the order parameter from Fig. 2(a), but now on a log-log scale. The high-temperature background (averaged between 40 and $50 \mathrm{~K}$ ) is indicated by the horizontal dashed line, while superconducting $T_{c}$ is indicated by the vertical dashed line. An inflection point at $T_{c}$ is clear.

In addition, we have modeled the strong upturn in the elastic order parameter for $x=0.19$ at low temperatures using a phenomenological expression

$$
A(T)=a+b T^{-\alpha}
$$

for temperatures $\leqslant 12 \mathrm{~K}$, as shown by the dashed red line in the inset of Fig. 9. We then phenomenologically decompose our measured order parameter (OP) according to

$$
\mathrm{OP}(T)=A(T) \times 2 \operatorname{DOP}(T),
$$

which gives the two-dimensional order parameter (2DOP) for our $x=0.19$ sample in the inset of Fig. 9. This decomposition has the interpretation that the effect of the $\mathrm{Cu}^{2+}-\mathrm{Nd}^{3+}$ coupling is to amplify the preexisting $2 \mathrm{D} \mathrm{Cu}^{2+}$ order parameter at low temperatures. While it is phenomenological, it reproduces in the elastic order parameter similar plateau behavior to that seen in the temperature dependence of $\chi^{\prime \prime}(Q, \hbar \omega=2 \mathrm{meV})$, shown in Fig. 8.

Such intriguing plateaus as a function of decreasing temperature in the magnetic Bragg intensity (the order parameter) and the low-energy spectral weight of the spin fluctuations have been previously observed in several heavy fermion

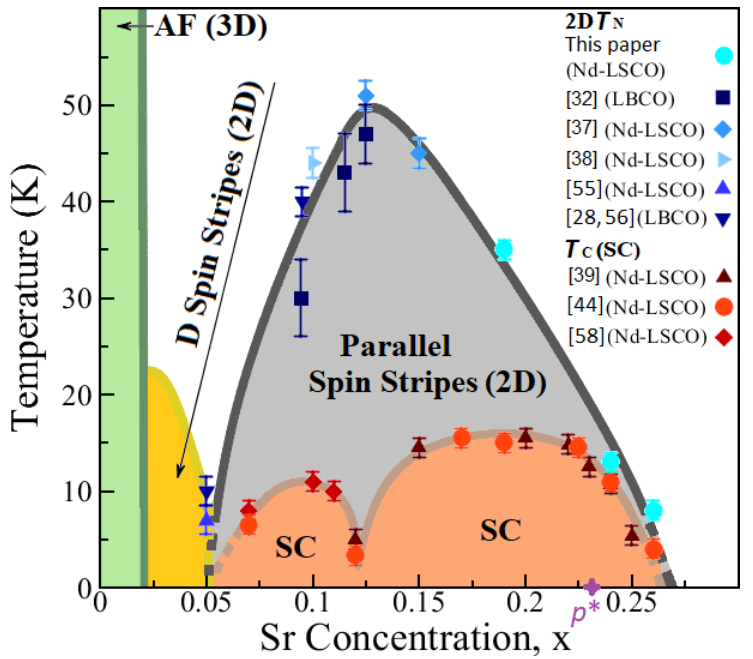

FIG. 10. Phase diagram for quasistatic magnetism in Nd-LSCO as measured by neutron scattering. Combining the current TAS data for $2 \mathrm{D} T_{N}$ with preexisting Nd-LSCO data, and complemented with LBCO results for $0.05 \leqslant x \leqslant 0.125$, we extend the regime of a $2 \mathrm{D}$ parallel spin stripe phase to $0.05 \leqslant x \leqslant 0.26$. Such a $2 \mathrm{D}$ parallel spin stripe phase is then a prerequisite for superconductivity. All 2D $T_{N}$ parallel spin stripe data come from elastic neutron scattering studies of either Nd-LSCO or LBCO as indicated in the legend. Superconducting $T_{c}$ for Nd-LSCO are also from references indicated in the legend. $p^{*}$ indicates the quantum critical point associated with the pseudogap phase [39]. SC, superconductivity.

superconductors, for example, $\mathrm{UPt}_{3}[51,52]$ and $\mathrm{UPd}_{2} \mathrm{Al}_{3}$ [53]. Anomalies in the low-energy spectral weight of spin fluctuations have also been observed in the LSCO cuprate system [54]. The coincidence of the plateaus with the superconducting $T_{c}$ was taken as evidence for strong coupling between magnetism and superconductivity and a key role for AF spin fluctuations in mediating superconductivity in these highly correlated, heavy fermion metals.

\section{PHASE DIAGRAM AND DISCUSSION}

Our measurements of 2D $T_{N}$ and the onset of 2D parallel stripes at optimal and high hole-doping levels in Nd-LSCO, with $x=0.19,0.24$, and 0.26 , allow us to complete the phase diagram for $2 \mathrm{D}$ parallel stripes and to examine their relation to superconductivity. These results extend earlier measurements for Nd-LSCO with $x \leqslant 0.15$ [37,55]. We have complemented these Nd-LSCO data with corresponding results from LBCO for $0.05 \leqslant x \leqslant 0.125[28,32,56]$. LBCO is a useful proxy for Nd-LSCO at these low doping values, as both LBCO and Nd-LSCO show very pronounced " $\frac{1}{8}$ anomalies" with superconducting $T_{c} \mathrm{~s}$ suppressed to $\sim 3 \mathrm{~K}$ [57]. In addition, the onset temperature for 2D parallel stripes in both $\mathrm{LBCO}$ and Nd-LSCO are maximal at $x=0.125$, with similar 2D $T_{N} \mathrm{~s} \sim 50 \mathrm{~K}[32,37]$.

Figure 10 shows the phase diagram for 2D parallel stripes in Nd-LSCO, superposed with its phase diagram for superconductivity. The superconducting transition temperatures are taken from earlier work $[39,44,58]$ and include data for the four single-crystal samples that were the subject of the present neutron scattering experiments, $x=0.125,0.19,0.24$, 
and 0.26 [44]. As previously mentioned, the superconducting phase behavior shows an onset of superconducting ground states at $x=0.05$, which coincides with the rotation of the 2D diagonal spin stripe structure to the 2D parallel spin stripe structure. Optimal superconducting $T_{c} \sim 15 \mathrm{~K}$ is reached for Nd-LSCO at $x \sim 0.19$, and the end of the superconducting dome occurs near $x \sim 0.27$ [44].

We can see that the entire region of superconducting ground states for Nd-LSCO is contained within the 2D parallel stripe phase. Superconductivity begins and ends coincident with the beginning and ending of the 2D static parallel stripe phase, as a function of concentration. At a given hole-doping level, superconductivity is entered from a state with static $2 \mathrm{D}$ parallel stripe order, as evidenced by quasi-Bragg peaks, and the quasi-Bragg peaks coexist with superconductivity below $\mathrm{T}_{c}$.

The superconducting $T_{c}$ vs $x$ relationship in Nd-LSCO is structured over this range of concentrations, $0.05 \leqslant x \leqslant 0.27$, due to the $\frac{1}{8}$ anomaly at $x=0.125$. Hence the strongest 2D parallel spin stripe order, in terms of the highest $2 \mathrm{D} T_{N}$, appears anticorrelated with superconductivity. However, parallel charge stripes have their onset at temperatures higher still than 2D $T_{N}$ and are intertwined with the parallel spin stripes at and near $x=0.125$. The charge stripes have been extensively studied in LBCO and to a somewhat lesser extent in NdLSCO, but less so still in LSCO_presumably because they are weaker still. The suppression of $T_{c}$ due to the $\frac{1}{8}$ anomaly in LSCO is itself much less pronounced than in either Nd-LSCO or LBCO. One could therefore argue that the conditions for enhanced superconductivity in these single-layer, hole-doped cuprates is the presence of 2D parallel spin stripes, without, or with less well developed, parallel charge stripes.

\section{CONCLUSION}

Recent NMR and ultrasound experiments at high magnetic field in LSCO have reported glassy antiferromagnetism existing to higher doping levels than previously believed, up to $\sim 0.19$ [59]. These measurements require a strong magnetic field to quench superconductivity which would otherwise hide the glassy antiferromagnetism in these experiments. As the enigmatic pseudogap phase in LSCO $[15,16,60]$ itself exists up to $p^{*} \sim 0.19$, this result implies that strong AF correlations dominate the entire pseudogap phase in LSCO but do not extend beyond $p^{*}$.

The pseudogap phase is also well studied in the Nd-LSCO system, and recent work has shown strong thermodynamic evidence for a pseudogap quantum critical point at $p^{*} \sim$ 0.23 [39]. Therefore the corresponding argument in NdLSCO would have static AF correlations existing to even higher hole-doping levels than in LSCO, such as $p^{*} \sim 0.23$ in Nd-LSCO. Such a picture of magnetism being restricted to $p<p^{*}$ would have been consistent with the previously measured, relatively weak order parameter for Nd-LSCO, $x=0.20$ [47], shown in Fig. 3. However, it is not consistent with our order parameter results on Nd-LSCO with $x=$ $0.19,0.24$, and 0.26 , all of which clearly show quasi-Bragg peaks due to static parallel spin stripe correlations at low temperatures.
Nonetheless, these NMR and ultrasound results in LSCO [59] are broadly consistent with the present observation of parallel spin stripes existing in Nd-LSCO over the concentration range $0.05 \leqslant x \leqslant 0.26$, in that they both show evidence for static parallel spin stripe order to higher doping than previously thought. In addition, while $x=0.26$ is clearly higher than $p^{*} \sim 0.23$, elastic neutron scattering measures "static" correlations at much shorter time scales than does NMR $\left(10^{-10} \mathrm{~s}\right.$ for neutrons compared with $10^{-6} \mathrm{~s}$ for NMR). Consequently, spin correlations on time scales falling between neutron "static" and NMR "static" would appear static to neutrons and dynamic to NMR. One may therefore expect to observe static antiferromagnetism with neutron scattering across a broader range of doping than with NMR.

There remains the question of how the pseudogap quantum critical point at $p^{*} \sim 0.23$ in Nd-LSCO manifests itself in the 2D static parallel spin stripe magnetism that is the subject of this paper. It is not through the nature of the quasistatic spin stripe order, which evolves smoothly across the four NdLSCO samples studied here, and therefore smoothly across $p^{*}$. Indeed, in and of itself, this is an important result as it decouples quasistatic parallel spin stripe magnetism from the pseudogap's quantum critical point at $p^{*}$. However, as already mentioned, the story for low-energy parallel spin stripe fluctuations is different. Figures 6 and 7 show the spectral weight of the parallel spin stripe fluctuations for $\hbar \omega<3 \mathrm{meV}$ at $x=0.19$ to be qualitatively different from that associated with samples with $x>p^{*} \sim 0.23: x=0.24$ and 0.26 . These low-energy inelastic parallel spin stripe fluctuations are much stronger and more strongly peaked at the IC ordering wave vector at $x=0.19$ than at $x=0.24$ and 0.26 . The temperature scale for the crossover or phase transition associated with the pseudogap phase is itself known to be much higher than either 2D $T_{N}$ or superconducting $T_{c}$ in Nd-LSCO [44]. For example, this $T^{*}$ is $\sim 100 \mathrm{~K}$ at $x=0.19$, and it would seem more reasonable to associate the pseudogap with the relatively high energy scale of these parallel spin stripe fluctuations, rather than the low energy scale of the quasistatic parallel spin stripe magnetism.

\section{ACKNOWLEDGMENTS}

We thank Louis Taillefer, Amireza Ataei, Takashi Imai, and Graeme Luke for useful and stimulating discussions. We also thank Jianshi Zhou and Zongyao Li for valuable insights into the crystal growth of Nd-LSCO. This work was supported by the Natural Sciences and Engineering Research Council of Canada. We acknowledge the access to neutron infrastructure and supporting technical assistance from the Australian Centre for Neutron Scattering. This research used resources at the Spallation Neutron Source and the High Flux Isotope Reactor, DOE Office of Science User Facilities, operated by the Oak Ridge National Laboratory (ORNL).

Q.M., K.C.R., and B.D.G. performed and analyzed the single-crystal TAS experiment on Nd-LSCO $x=0.19$ and 0.26 at TAIPAN, ANSTO. Q.M., Z.W.C., S.C., and B.D.G. performed and analyzed the TAS experiment on Nd-LSCO $x=0.24$ at HB3, HFIR. Q.M., A.I.K., M.B.S., E.M.S., and B.D.G performed and analyzed the TOF scattering 
experiments on Nd-LSCO $x=0.125,0.19$, and 0.24 at SEQUOIA, SNS. Q.M., M.D., and G.M. carried out the material synthesis and crystal growth. B.D.G. and Q.M. wrote the manuscript, while B.D.G. conceived and oversaw the project.
[1] J. G. Bednorz and K. A. Müller, Possible high $T_{c}$ superconductivity in the Ba-La-Cu-O system, Z. Phys. B: Condens. Matter 64, 189 (1986).

[2] M. K. Wu, J. R. Ashburn, C. J. Torng, P. H. Hor, R. L. Meng, L. Gao, Z. J. Huang, Y. Q. Wang, and C. W. Chu, Superconductivity at $93 \mathrm{~K}$ in a New Mixed-Phase Y-Ba-Cu-O Compound System at Ambient Pressure, Phys. Rev. Lett. 58, 908 (1987).

[3] P. W. Anderson, The resonating valence bond state in $\mathrm{La}_{2} \mathrm{CuO}_{4}$ and superconductivity, Science 235, 1196 (1987).

[4] V. J. Emery, Theory of High- $\mathrm{T}_{\mathrm{c}}$ Superconductivity in Oxides, Phys. Rev. Lett. 58, 2794 (1987).

[5] L. C. Bourne, A. Zettl, T. W. Barbee III, and M. L. Cohen, Complete absence of isotope effect in $\mathrm{YBa}_{2} \mathrm{Cu}_{3} \mathrm{O}_{7}$ : Consequences for phonon-mediated superconductivity, Phys. Rev. B 36, 3990 (1987).

[6] G. Kotliar and J. Liu, Superexchange mechanism and $d$-wave superconductivity, Phys. Rev. B 38, 5142 (1988).

[7] J. R. Schrieffer, X.-G. Wen, and S.-C. Zhang, Spin-Bag Mechanism of High-Temperature Superconductivity, Phys. Rev. Lett. 60, 944 (1988).

[8] F. C. Zhang and T. M. Rice, Effective Hamiltonian for the superconducting Cu oxides, Phys. Rev. B 37, 3759 (1988).

[9] J. P. Franck, Experimental studies of the isotope effect in high temperature superconductors, in Physical Properties of High Temperature Superconductors IV, edited by D. M. Ginsberg (World Scientific, Singapore, 1994), Chap. 4, pp. 189-293.

[10] V. J. Emery, S. A. Kivelson, and O. Zachar, Spin-gap proximity effect mechanism of high-temperature superconductivity, Phys. Rev. B 56, 6120 (1997).

[11] M. L. Kulić, Interplay of electron-phonon interaction and strong correlations: the possible way to high-temperature superconductivity, Phys. Rep. 338, 1 (2000).

[12] P. A. Lee, N. Nagaosa, and X.-G. Wen, Doping a Mott insulator: Physics of high-temperature superconductivity, Rev. Mod. Phys. 78, 17 (2006).

[13] H. Mukuda, S. Shimizu, A. Iyo, and Y. Kitaoka, High- $T_{c}$ superconductivity and antiferromagnetism in multilayered copper oxides-a new paradigm of superconducting mechanism-, J. Phys. Soc. Jpn. 81, 011008 (2011).

[14] D. J. Scalapino, A common thread: The pairing interaction for unconventional superconductors, Rev. Mod. Phys. 84, 1383 (2012).

[15] L. Taillefer, Scattering and pairing in cuprate superconductors, Annu. Rev. Condens. Matter Phys. 1, 51 (2010).

[16] C. Proust and L. Taillefer, The remarkable underlying ground states of cuprate superconductors, Annu. Rev. Condens. Matter Phys. 10, 409 (2019).

[17] B. Keimer, S. A. Kivelson, M. R. Norman, S. Uchida, and J. Zaanen, From quantum matter to high-temperature superconductivity in copper oxides, Nature (London) 518, 179 (2015).

[18] P. Corboz, T. M. Rice, and M. Troyer, Competing States in the $t$ $J$ Model: Uniform $d$-Wave State versus Stripe State, Phys. Rev. Lett. 113, 046402 (2014).
[19] V. Thampy, M. P. M. Dean, N. B. Christensen, L. Steinke, Z. Islam, M. Oda, M. Ido, N. Momono, S. B. Wilkins, and J. P. Hill, Rotated stripe order and its competition with superconductivity in $\mathrm{La}_{1.88} \mathrm{Sr}_{0.12} \mathrm{CuO}_{4}$, Phys. Rev. B 90, 100510(R) (2014).

[20] B. Keimer and J. E. Moore, The physics of quantum materials, Nat. Phys. 13, 1045 (2017).

[21] M. A. Kastner, R. J. Birgeneau, G. Shirane, and Y. Endoh, Magnetic, transport, and optical properties of monolayer copper oxides, Rev. Mod. Phys. 70, 897 (1998).

[22] B. Keimer, N. Belk, R. J. Birgeneau, A. Cassanho, C. Y. Chen, M. Greven, M. A. Kastner, A. Aharony, Y. Endoh, R. W. Erwin, and G. Shirane, Magnetic excitations in pure, lightly doped, and weakly metallic $\mathrm{La}_{2} \mathrm{CuO}_{4}$, Phys. Rev. B 46, 14034 (1992).

[23] J. H. Cho, F. C. Chou, and D. C. Johnston, Phase Separation and Finite Size Scaling in $\mathrm{La}_{2-x} \mathrm{Sr}_{x} \mathrm{CuO}_{(4+\delta)}[0<(x, \gamma)<0.03]$, Phys. Rev. Lett. 70, 222 (1993).

[24] F. C. Chou, F. Borsa, J. H. Cho, D. C. Johnston, A. Lascialfari, D. R. Torgeson, and J. Ziolo, Magnetic Phase Diagram of Lightly Doped $\mathrm{La}_{2-x} \mathrm{Sr}_{x} \mathrm{CuO}_{4}$ from ${ }^{139} \mathrm{La}$ Nuclear Quadrupole Resonance, Phys. Rev. Lett. 71, 2323 (1993).

[25] F. Borsa, P. Carretta, J. H. Cho, F. C. Chou, Q. Hu, D. C. Johnston, A. Lascialfari, D. R. Torgeson, R. J. Gooding, N. M. Salem, and K. J. E. Vos, Staggered magnetization in $\mathrm{La}_{2-x} \mathrm{Sr}_{x} \mathrm{CuO}_{4}$ from ${ }^{139} \mathrm{La} \mathrm{NQR}$ and $\mu$ SR: Effects of Sr doping in the range $0<x<0.02$, Phys. Rev. B 52, 7334 (1995).

[26] S. Wakimoto, R. J. Birgeneau, M. A. Kastner, Y. S. Lee, R. Erwin, P. M. Gehring, S. H. Lee, M. Fujita, K. Yamada, Y. Endoh, K. Hirota, and G. Shirane, Direct observation of a one-dimensional static spin modulation in insulating $\mathrm{La}_{1.95} \mathrm{Sr}_{0.05} \mathrm{CuO}_{4}$, Phys. Rev. B 61, 3699 (2000).

[27] S. Wakimoto, G. Shirane, Y. Endoh, K. Hirota, S. Ueki, K. Yamada, R. J. Birgeneau, M. A. Kastner, Y. S. Lee, P. M. Gehring, and S. H. Lee, Observation of incommensurate magnetic correlations at the lower critical concentration for superconductivity in $\mathrm{La}_{2-x} \mathrm{Sr}_{x} \mathrm{CuO}_{4}(x=0.05)$, Phys. Rev. B 60, R769 (1999).

[28] S. R. Dunsiger, Y. Zhao, B. D. Gaulin, Y. Qiu, P. Bourges, Y. Sidis, J. R. D. Copley, A. Kallin, E. M. Mazurek, and H. A. Dabkowska, Diagonal and collinear incommensurate spin structures in underdoped $\mathrm{La}_{2-x} \mathrm{Ba}_{x} \mathrm{CuO}_{4}$, Phys. Rev. B 78, 092507 (2008).

[29] M. Fujita, H. Goka, K. Yamada, J. M. Tranquada, and L. P. Regnault, Stripe order, depinning, and fluctuations in $\mathrm{La}_{1.875} \mathrm{Ba}_{0.125} \mathrm{CuO}_{4}$ and $\mathrm{La}_{1.875} \mathrm{Ba}_{0.075} \mathrm{Sr}_{0.050} \mathrm{CuO}_{4}$, Phys. Rev. B 70, 104517 (2004)

[30] K. Yamada, C. H. Lee, K. Kurahashi, J. Wada, S. Wakimoto, S. Ueki, H. Kimura, Y. Endoh, S. Hosoya, G. Shirane, R. J. Birgeneau, M. Greven, M. A. Kastner, and Y. J. Kim, Doping dependence of the spatially modulated dynamical spin correlations and the superconducting-transition temperature in $\mathrm{La}_{2-x} \mathrm{Sr}_{x} \mathrm{CuO}_{4}$, Phys. Rev. B 57, 6165 (1998).

[31] M. Kofu, S.-H. Lee, M. Fujita, H.-J. Kang, H. Eisaki, and K. Yamada, Hidden Quantum Spin-Gap State in the Static Stripe 
Phase of High-Temperature $\mathrm{La}_{2-x} \mathrm{Sr}_{x} \mathrm{CuO}_{4}$ Superconductors, Phys. Rev. Lett. 102, 047001 (2009).

[32] M. Hücker, M. v. Zimmermann, G. D. Gu, Z. J. Xu, J. S. Wen, G. Xu, H. J. Kang, A. Zheludev, and J. M. Tranquada, Stripe order in superconducting $\mathrm{La}_{2-x} \mathrm{Ba}_{x} \mathrm{CuO}_{4}(0.095 \leqslant x \leqslant 0.155)$, Phys. Rev. B 83, 104506 (2011).

[33] B. Nachumi, Y. Fudamoto, A. Keren, K. M. Kojima, M. Larkin, G. M. Luke, J. Merrin, O. Tchernyshyov, Y. J. Uemura, N. Ichikawa, M. Goto, H. Takagi, S. Uchida, M. K. Crawford, E. M. McCarron, D. E. MacLaughlin, and R. H. Heffner, Muon spin relaxation study of the stripe phase order in $\mathrm{La}_{1.6-x} \mathrm{Nd}_{0.4} \mathrm{Sr}_{x} \mathrm{CuO}_{4}$ and related 214 cuprates, Phys. Rev. B 58, 8760 (1998).

[34] P. M. Singer, A. W. Hunt, A. F. Cederström, and T. Imai, Systematic ${ }^{63} \mathrm{Cu}$ NQR Study of the Stripe Phase in $\mathrm{La}_{1.6-x} \mathrm{Nd}_{0.4} \mathrm{Sr}_{x} \mathrm{CuO}_{4}$ for $0.07<x<0.25$, Phys. Rev. B 60, 15345 (1999).

[35] A. W. Hunt, P. M. Singer, A. F. Cederström, and T. Imai, Glassy slowing of stripe modulation in $(\mathrm{La}, \mathrm{Eu}, \mathrm{Nd})_{2-x}(\mathrm{Sr}, \mathrm{Ba})_{x} \mathrm{CuO}_{4}$ : A ${ }^{63} \mathrm{Cu}$ and ${ }^{139} \mathrm{La} \mathrm{NQR}$ study down to $350 \mathrm{mK}$, Phys. Rev. B 64, 134525 (2001).

[36] J. M. Tranquada, B. J. Sternlieb, J. D. Axe, Y. Nakamura, and S. Uchida, Evidence for stripe correlations of spins and holes in copper oxide superconductors, Nature (London) 375, 561 (1995).

[37] J. M. Tranquada, J. D. Axe, N. Ichikawa, A. R. Moodenbaugh, Y. Nakamura, and S. Uchida, Coexistence of, and Competition between, Superconductivity and Charge-Stripe Order in $\mathrm{La}_{1.6-x} \mathrm{Nd}_{0.4} \mathrm{Sr}_{x} \mathrm{CuO}_{4}$, Phys. Rev. Lett. 78, 338 (1997).

[38] N. Ichikawa, S. Uchida, J. M. Tranquada, T. Niemöller, P. M. Gehring, S.-H. Lee, and J. R. Schneider, Local Magnetic Order vs Superconductivity in a Layered Cuprate, Phys. Rev. Lett. 85, 1738 (2000).

[39] B. Michon, C. Girod, S. Badoux, J. Kačmarčík, Q. Ma, M. Dragomir, H. A. Dabkowska, B. D. Gaulin, J.-S. Zhou, S. Pyon, T. Takayama, H. Takagi, S. Verret, N. Doiron-Leyraud, C. Marcenat, L. Taillefer, and T. Klein, Thermodynamic signatures of quantum criticality in cuprate superconductors, Nature (London) 567, 218 (2019).

[40] H. Takagi, T. Ido, S. Ishibashi, M. Uota, S. Uchida, and Y. Tokura, Superconductor-to-nonsuperconductor transition in $\left(\mathrm{La}_{1-x} \mathrm{Sr}_{x}\right)_{2} \mathrm{CuO}_{4}$ as investigated by transport and magnetic measurements, Phys. Rev. B 40, 2254 (1989).

[41] T. Suzuki, T. Goto, K. Chiba, T. Shinoda, T. Fukase, H. Kimura, K. Yamada, M. Ohashi, and Y. Yamaguchi, Observation of modulated magnetic long-range order in $\mathrm{La}_{1.88} \mathrm{Sr}_{0.12} \mathrm{CuO}_{4}$, Phys. Rev. B 57, R3229 (1998).

[42] T. P. Croft, C. Lester, M. S. Senn, A. Bombardi, and S. M. Hayden, Charge density wave fluctuations in $\mathrm{La}_{2-x} \mathrm{Sr}_{x} \mathrm{CuO}_{4}$ and their competition with superconductivity, Phys. Rev. B 89, 224513 (2014).

[43] H. Miao, G. Fabbris, R. J. Koch, D. G. Mazzone, C. S. Nelson, R. Acevedo-Esteves, Y. Li, G. D. Gu, T. Yilmaz, K. Kaznatcheev, E. Vescovo, M. Oda, K. Kurosawa, N. Momono, T. A. Assefa, I. K. Robinson, E. Bozin, J. M. Tranquada, P. D. Johnson, and M. P. M. Dean, Charge density waves in cuprate superconductors beyond the critical doping, npj Quantum Mater. 6, 31 (2021).

[44] M. Dragomir, Q. Ma, J. P. Clancy, A. Ataei, P. A. Dube, S. Sharma, A. Huq, H. A. Dabkowska, L. Taillefer, and B. D.
Gaulin, Materials preparation, single-crystal growth, and the phase diagram of the cuprate high-temperature superconductor $\mathrm{La}_{1.6-x} \mathrm{Nd}_{0.4} \mathrm{Sr}_{x} \mathrm{CuO}_{4}$, Phys. Rev. Material 4, 114801 (2020).

[45] R. Daou, N. Doiron-Leyraud, D. LeBoeuf, S. Y. Li, F. Laliberté, O. Cyr-Choiniere, Y. J. Jo, L. Balicas, J.-Q. Yan, J.-S. Zhou, J. B. Goodenough, and L. Taillefer, Linear temperature dependence of resistivity and change in the Fermi surface at the pseudogap critical point of a high- $T_{c}$ superconductor, Nat. Phys. 5, 31 (2009).

[46] G. E. Granroth, A. I. Kolesnikov, T. E. Sherline, J. P. Clancy, K. A. Ross, J. P. C Ruff, B. D. Gaulin, and S. E. Nagler, SEQUOIA: A newly operating chopper spectrometer at the SNS, J. Phys.: Conf. Ser. 251, 012058 (2010).

[47] J. M. Tranquada, N. Ichikawa, and S. Uchida, Glassy nature of stripe ordering in $\mathrm{La}_{1.6-x} \mathrm{Nd}_{0.4} \mathrm{Sr}_{x} \mathrm{CuO}_{4}$, Phys. Rev. B 59, 14712 (1999).

[48] J. M. Tranquada, J. D. Axe, N. Ichikawa, Y. Nakamura, S. Uchida, and B. Nachumi, Neutron-scattering study of stripephase order of holes and spins in $\mathrm{La}_{1.48} \mathrm{Nd}_{0.4} \mathrm{Sr}_{0.12} \mathrm{CuO}_{4}$, Phys. Rev. B 54, 7489 (1996).

[49] J. J. Wagman, G. Van Gastel, K. A. Ross, Z. Yamani, Y. Zhao, Y. Qiu, J. R. D. Copley, A. B. Kallin, E. Mazurek, J. P. Carlo, H. A. Dabkowska, and B. D. Gaulin, Two-dimensional incommensurate and three-dimensional commensurate magnetic order and fluctuations in $\mathrm{La}_{2-x} \mathrm{Ba}_{x} \mathrm{CuO}_{4}$, Phys. Rev. B 88, 014412 (2013).

[50] J. M. Tranquada, Neutron scattering studies of antiferromagnetic correlations in cuprates, in Handbook of HighTemperature Superconductivity (Springer, New York, 2007), pp. 257-298.

[51] G. Aeppli, E. Bucher, C. Broholm, J. K. Kjems, J. Baumann, and J. Hufnagl, Magnetic Order and Fluctuations in Superconducting $\mathrm{UP}_{3}$, Phys. Rev. Lett. 60, 615 (1988).

[52] E. D. Isaacs, P. Zschack, C. L. Broholm, C. Burns, G. Aeppli, A. P. Ramirez, T. T. M. Palstra, R. W. Erwin, N. Stücheli, and E. Bucher, Antiferromagnetism and its Relation to the Superconducting Phases of UPt $t_{3}$, Phys. Rev. Lett. 75, 1178 (1995).

[53] N. Metoki, Y. Haga, Y. Koike, N. Aso, and Y. Onuki, Coupling between magnetic and superconducting order parameters and evidence for the spin excitation gap in the superconducting state of a heavy fermion superconductor $\mathrm{UPd}_{2} \mathrm{Al}_{3}$, J. Phys. Soc. Jpn. 66, 2560 (1997).

[54] A. T. Rømer, J. Chang, N. B. Christensen, B. M. Andersen, K. Lefmann, L. Mähler, J. Gavilano, R. Gilardi, Ch. Niedermayer, H. M. Rønnow, A. Schneidewind, P. Link, M. Oda, M. Ido, N. Momono, and J. Mesot, Glassy low-energy spin fluctuations and anisotropy gap in $\mathrm{La}_{1.88} \mathrm{Sr}_{0.12} \mathrm{CuO}_{4}$, Phys. Rev. B 87, 144513 (2013)

[55] S. Wakimoto, J. M. Tranquada, T. Ono, K. M. Kojima, S. Uchida, S.-H Lee, P. M. Gehring, and R. J. Birgeneau, Diagonal static spin correlation in the low-temperature orthorhombic Pccn phase of $\mathrm{La}_{1.55} \mathrm{Nd}_{0.4} \mathrm{Sr}_{0.05} \mathrm{CuO}_{4}$, Phys. Rev. B 64, 174505 (2001).

[56] S. R. Dunsiger, Y. Zhao, Z. Yamani, W. J. L. Buyers, H. A. Dabkowska, and B. D. Gaulin, Incommensurate spin ordering and fluctuations in underdoped $\mathrm{La}_{2-x} \mathrm{Ba}_{x} \mathrm{CuO}_{4}$, Phys. Rev. B 77, 224410 (2008)

[57] J. D. Axe, A. H. Moudden, D. Hohlwein, D. E. Cox, K. M. Mohanty, A. R. Moodenbaugh, and Y. Xu, Structural Phase Transformations and Superconductivity in $\mathrm{La}_{2-\mathrm{x}} \mathrm{Ba}_{\mathrm{x}} \mathrm{CuO}_{4}$, Phys. Rev. Lett. 62, 2751 (1989). 
[58] J. D. Axe and M. K. Crawford, Structural instabilities in lanthanum cuprate superconductors, J. Low Temp. Phys. 95, 271 (1994).

[59] M. Frachet, I. Vinograd, R. Zhou, S. Benhabib, S. Wu, H. Mayaffre, S. Krämer, S. K. Ramakrishna, A. Reyes, J. Debray, T. Kurosawa, N. Momono, M. Oda, S. Komiya, S. Ono, M.Horio, J. Chang, C. Proust, D. LeBoeuf, and M.-H. Julien,
Hidden magnetism at the pseudogap critical point of a cuprate superconductor, Nat. Phys. 16, 1064 (2020).

[60] P. Giraldo-Gallo, J. A. Galvis, Z. Stegen, K. A Modic, F. F. Balakirev, J. B. Betts, X. Lian, C. Moir, S. C. Riggs, J. Wu, A. T. Bollinger, X. He, I. Božovi, B. J. Ramshaw, R. D. McDonald, G. S. Boebinger, and A. Shekhter, Scale-invariant magnetoresistance in a cuprate superconductor, Science 361, 479 (2018). 